ARTIGO ORIGINAL

\title{
Dimensão de parcelas para levantamento da vegetação arbórea regenerante em um fragmento de Floresta Atlântica em Pernambuco, Brasil
}

\author{
Dimension of plots for inventory of regenerating tree vegetation in a fragment of \\ Atlantic Forest in Pernambuco, Brazil \\ Nelio Domingos da Silva ${ }^{1}$ (D), Luiz Carlos Marangon ${ }^{1}$, , Ana Lícia Patriota Feliciano Marangon (D), \\ José Antônio Aleixo da Silva' ${ }^{1}$ (1) \\ ${ }^{1}$ Departamento de Ciência Florestal, Universidade Federal Rural de Pernambuco - UFRPE. Recife, PE, Brasil
}

Como citar: Silva, N. D., Marangon, L. C., Marangon, A. L. P. F., \& Silva, J. A. A. (2020). Dimensão de parcelas para levantamento da vegetação arbórea regenerante em um fragmento de Floresta Atlântica em Pernambuco, Brasil. Scientia Forestalis, 48(127), e3091. https://doi.org/10.18671/scifor.v48n127.05

\begin{abstract}
Resumo
Este estudo avalia o método da eficiência relativa da amostragem na definição de dimensão de parcelas em levantamentos de espécies arbóreas regenerantes em um fragmento de Floresta Atlântica em Pernambuco, Brasil. Foram instaladas 40 parcelas para cada uma das diferentes dimensões analisadas. Todos os indivíduos com altura $\geq 1,0$ metro e circunferência à altura do peito a 1,30m do solo $<15,0 \mathrm{~cm}$ foram catalogados, identificados e mensurados. Foram calculadas as suficiências amostrais e florística, curvas de rarefação e estimativas de Jackknife, assim como a eficiência relativa para as de $5 \mathrm{~m} \times 10 \mathrm{~m}, 10 \mathrm{~m}$ $\times 10 \mathrm{~m}$ e $10 \mathrm{~m} \times 25 \mathrm{~m}$ por satisfazerem o critério de erro amostral admissível. A amostragem da vegetação arbórea regenerante indicou que a parcela de $10 \mathrm{~m} \times 10 \mathrm{~m}$ é a mais eficiente dada a elevada precisão amostral, alta riqueza de espécies e baixo tempo de amostragem.
\end{abstract}

Palavras-chave: Suficiência amostral; Regeneração natural; Floresta Ombrófila Densa.

\begin{abstract}
This study aims to assess the methods of relative efficiency of the sampling in the definition of the area and shape of plots in surveys of regenerating tree in a fragment of the Atlantic Forest in Pernambuco, Brazil. 40 plots were installed for each of the different dimensions analyzed. All individuals with height $\geq$ 1.0 meter and circumference at breast height $(1.30 \mathrm{~m}$ above of ground) $<15.0 \mathrm{~cm}$ were cataloged, identified and measured. The sampling and floristic sufficiency, rarefaction curves, Jackknife estimates and the relative efficiency were calculated and plotted for the plots dimensions of $5 \mathrm{~m} \times 10 \mathrm{~m}, 10 \mathrm{~m} \times 10 \mathrm{~m}$ and $10 \mathrm{~m} \times 25 \mathrm{~m}$ because they satisfied the sampling error criterion admissible. The sampling of regenerating tree vegetation indicated that the plot of $10 \mathrm{~m} \times 10 \mathrm{~m}$ is the most efficient given the high sampling precision, high species richness and low sampling time.
\end{abstract}

Keywords: Sampling sufficiency; Natural regeneration; Dense Ombrophylous Forest.

\section{INTRODUÇÃO}

Em decorrência da fragmentação de habitats, a biodiversidade registrada nos remanescentes florestais atlânticos de Pernambuco vem sofrendo consequências negativas tanto do efeito causado pela redução da área original, como da interação entre a borda da floresta e a matriz circundante (Oliveira et al., 2015).

Fonte de financiamento: Coordenação de Aperfeiçoamento de Pessoal de Nível Superior (CAPES)

Conflito de interesse: Nada a declarar

Autor correspondente: nelio.domingos@gmail.com

Recebido: 28 setembro 2018

Aceito: 06 agosto 2019.

Editor: Paulo Henrique Müller Silva.

(c) Este é um artigo publicado em acesso aberto (Open Access) sob a licença Creative Commons Attribution, que permite uso, distribuição e reprodução em qualquer meio, sem restrições desde que o trabalho original seja corretamente citado. 
Rode et al. (2010) fundamentam que a implementação de pesquisas que visem melhor compreender como as espécies se comportam estruturalmente diante dos distúrbios acarretados pela fragmentação florestal são importantes para avaliar a situação atual de conservação que estes remanescentes se encontram, assim como consolidar informações que facilitarão a adoção de políticas públicas, ações e medidas de conservação e restauração das florestas.

Os poucos trabalhos realizados na Floresta Atlântica do Estado de Pernambuco que incluem plantas no estádio juvenil, ou seja, aquelas que compõem parte do sub-bosque, geralmente utilizam unidades amostrais de $5 \mathrm{~m} \times 5 \mathrm{~m}$ no intuito de elucidar questões relativas ao potencial regenerativo da floresta (Silva et al., 2010; Torres, 2014; Lima, 2017; Silva, 2017).

Apesar de não existir um sistema de amostragem que seja aplicado universalmente, em estudos com espécies regenerantes na Floresta Atlântica devem-se levar em consideração as particularidades de cada fragmento florestal para obtenção de resultados confiáveis. Pois, cada fragmento apresenta características específicas de número de indivíduos e espécies, área basal, altura média, densidade total e idade, que influenciam diretamente na escolha da dimensão das unidades amostrais utilizadas (Péllico Neto \& Brena, 1997).

De acordo com Higuchi et al. (1982) a eficiência relativa é um indicador que analisa o tempo de um determinado método de amostragem, assim como sua precisão com base no coeficiente de variação e indica valores que determinarão quão eficientes será o método em comparação com outro. E a precisão amostral refere-se ao tamanho dos desvios da amostra em relação à média estimada, sendo obtida por intermédio da repetição do procedimento de amostragem (Bonetes, 2003).

Logo, em estudos com espécies regenerantes na Floresta Atlântica, a dimensão de parcela adotada influencia no tempo de mensuração, na precisão e na eficiência do inventário. Neste sentido, Meunier et al. (2001) destacam que a dimensão ideal de parcela é aquela que indica elevada riqueza, alto nível de precisão com tempo de amostragem reduzido.

Sendo assim, este trabalho tem como objetivo avaliar a eficiência relativa na definição da dimensão de parcelas em levantamentos de espécies arbóreas regenerantes em um fragmento de Floresta Ombrófila Densa das Terras Baixas em Jaboatão dos Guararapes, Pernambuco, Brasil.

\section{MATERIAL E MÉTODOS}

\section{Área de estudo}

O estudo foi realizado no fragmento de Floresta Ombrófila Densa das Terras Baixas, denominado Mata de Manassu. O fragmento florestal de 410,17ha está localizado na região norte da cidade de Jaboatão dos Guararapes, Pernambuco. Situa-se entre $8^{\circ} 04^{\prime} 44,5^{\prime \prime}$ de latitude Sul e $35^{\circ} 01^{\prime} 23,0^{\prime \prime}$ de longitude Oeste. Os solos são classificados em Latossolos Amarelo, Podzólicos Vermelho-Amarelos e Gleissolos, e apresenta relevo ondulado a forte ondulado (Silva et al., 2001).

As espécies arbóreas mais comuns na área são: Eschweilera ovata (Cambess.) Mart. Ex Miers, Psychotria carthagenensis Jacq., Cordia nodosa Lam., Erythroxylum mucronatum Benth., Siparuna guianensis Aubl., Hirtella racemosa Lam., Miconia prasina (Sw.) DC., Thyrsodium spruceanum Benth., Brosimum guianense (Aubl.) Huber e Erythroxylum squamatum Sw..

Segundo a classificação de Alvares et al. (2013) o clima da região é do tipo Am, ou seja, tropical de monção com estação chuvosa adiantada para o outono, precipitação anual acumulada de $1.487,2 \mathrm{~mm}$ e temperatura média em torno de $24^{\circ} \mathrm{C}$ (Agência Pernambucana de Águas e Clima, 2017).

\section{Coleta dos dados}

Foram implantadas unidades amostrais em dois ambientes: borda e interior. Para a borda foi considerada a extensão de até $100 \mathrm{~m}$ do limite florestal em direção ao interior e 
para o interior foi considerada a área com mais de $300 \mathrm{~m}$ de distância do limite florestal (Gomes et al., 2009).

Ao todo foram instaladas 40 parcelas de $10 \mathrm{~m} \times 25 \mathrm{~m}$, sendo 20 em cada ambiente, abrangendo $10.000 \mathrm{~m}^{2}$ de área amostral total (parcela $\left.\mathrm{D}\right)$. No vértice direito de cada parcela da borda e do interior do fragmento foram alocadas parcelas de: $5 \mathrm{~m} \times 5 \mathrm{~m}\left(1.000 \mathrm{~m}^{2}\right)$ (parcela A), $5 \mathrm{~m} \times 10 \mathrm{~m}\left(2.000 \mathrm{~m}^{2}\right)\left(\right.$ parcela B) e $10 \mathrm{~m} \times 10 \mathrm{~m}\left(4.000 \mathrm{~m}^{2}\right.$ ) (parcela C) (Figura 1), a fim de verificar a dimensão de parcela que apresenta melhor eficiência relativa para amostragem das variáveis (número de espécies e de indivíduos) da vegetação arbórea regenerante. Isto é, que apresente maior nível de informações ecológicas, por tempo de mensuração do inventário e elevado nível de precisão.

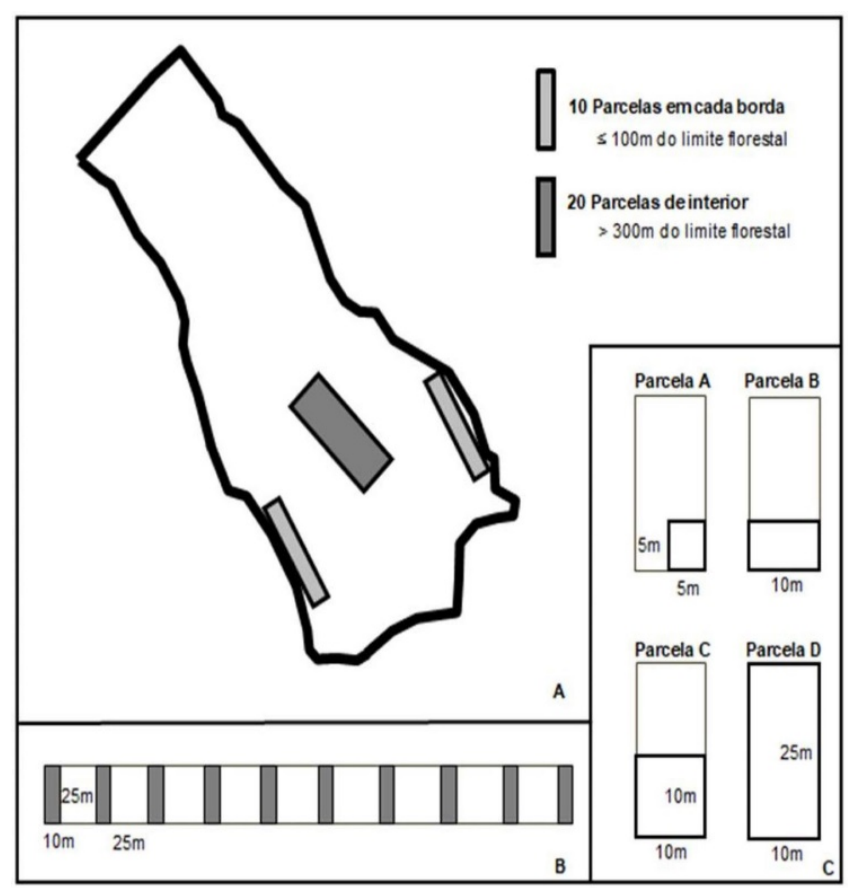

Figura 1. Esquema de distribuição das parcelas em ambientes de borda e interior do fragmento florestal (A); Espaçamentos entre as parcelas (B); Dimensões das parcelas (C).

As parcelas da borda foram alocadas ao longo de transectos paralelos as margens do limite do fragmento e equidistantes entre si em 25m, com 10 parcelas cada. No ambiente de interior lançou-se 20 parcelas de forma sistemática constituindo uma malha, na qual as parcelas foram espaçadas $25 \mathrm{~m}$ umas das outras.

No interior das parcelas foram mensuradas, com auxílio de fita métrica, as circunferências à altura da base $\left(\mathrm{CAB}_{0,30 \mathrm{~m})}\right.$ dos indivíduos com altura $\geq 1,0 \mathrm{~m}$ e com circunferência à altura do peito $\left(\mathrm{CAP}_{1,30} \mathrm{~m}\right)<15,0 \mathrm{~cm}$, assim como estimadas as respectivas alturas com o auxílio de uma tesoura de alta poda, com módulos de $2 \mathrm{~m}$.

Todas as unidades amostrais foram georreferenciadas com o auxílio do receptor GPS (Global Position System) Garmin modelo 76map CSx e demarcadas com o auxílio de fita métrica, piquetes de cano de PVC e cordão de nylon. Todos os indivíduos registrados receberam uma plaqueta de campo numerada em ordem crescente.

Durante as idas a campo foram anotados na ficha de campo, os seguintes dados: número de identificação do indivíduo na parcela; sua identificação botânica, data da amostragem, tempo total de mensuração dos indivíduos de cada parcela, além da coleta de material fértil, quando possível.

O material botânico coletado foi herborizado conforme as técnicas de preparação, secagem e montagem de exsicatas. As espécies foram identificadas de acordo com Angiosperm Phylogeny Group IV (Angiosperm Phylogeny Group IV, 2016) e consulta aos 
Herbários Professor Vasconcelos Sobrinho e Sérgio Tavares, ambos da Universidade Federal Rural de Pernambuco. A atual nomenclatura das espécies foi conferida por meio de consultas ao banco de dados do Missouri Botanical Garden (http://www.mobot.org) e do Tropicos (http://www.tropicos.org). Foram coletadas amostras botânicas férteis de ramos com flores e frutos, às quais foram introduzidas no Herbário Sérgio Tavares sob os números HST 21782 a HST 21834.

\section{Análise dos dados}

\section{Suficiência amostral}

Para comprovar que as amostras foram representativas em termos de número de indivíduos e de espécies em todas as dimensões de parcelas, no nível de $5 \%$ de probabilidade, realizou-se os cálculos dos erros amostrais ( $\mathrm{Ea} \%$ ) comparado com o erro adotado de $10 \%$.

\section{Dimensão de parcela}

Para verificar a dimensão de parcela que apresenta maior precisão amostral para as variáveis analisadas, foi realizado o cálculo do erro amostral ( $E a \%$ ), com erro adotado de $10 \%$ e nível de confiança de 95\%, conforme Fórmula (1) adaptada de Soares et al. (2011).

Fórmula: $E a \%= \pm \frac{\bar{y}_{y}}{\bar{Y}} * t * 100$

Em que:

$\bar{s}_{y}=$ Erro padrão da média;

$t=$ valor tabelado de t de "Student" com nível de 10\% de probabilidade de erro;

$\bar{Y}=$ média.

Foram realizados os cálculos de eficiência relativa para cada dimensão de parcela testada em relação à parcela considerada padrão para este estudo (parcela de $5 \mathrm{~m} \times 5 \mathrm{~m}$ ), ou seja, teoricamente portadora de uma eficiência relativa de $100 \%$.

O teste da eficiência relativa considera diferentes tamanhos de amostras associado a outras variáveis que são correlacionadas com estes, não favorecendo nem prejudicando qualquer tamanho de parcela na amostra (Meunier et al., 2001).

Para tal procedimento foi adaptada à Fórmula (2) proposta por Vasconcelos (1990):

Fórmula: $E R=\frac{(E A p) *(N p) *(\bar{T} p)}{(E A t) *(N t) *(\bar{T} t)}$

Em que:

$E A p=$ Erro amostral proposto;

$E A t=$ Erro amostral testado;

$N p$ = Tamanho da amostra proposta;

$N t$ = Tamanho da amostra testada;

$\bar{T} p=$ Tempo médio de mensuração por parcela proposta;

$\overline{T t}=$ Tempo médio de mensuração por parcela testada;

$E R=$ Eficiência relativa, em que $E R<1$ indica que a parcela padrão (com dimensão de $5 \mathrm{~m} \times 5 \mathrm{~m}$ ) é mais eficiente que a testada; se $E R \cong 1$ sugerem que ambas as dimensões de parcelas utilizadas são igualmente eficientes para a variável analisada; e se $E R>1$, indica que o uso da dimensão de parcela testada é mais eficiente quando comparada com a parcela padrão.

Para contabilização do tempo total de mensuração por dimensão de parcela, foram considerados os tempos de lançamento de cada unidade amostral investigada, acrescido dos 
tempos de mensuração de todos os indivíduos que atendiam os critérios de inclusão adotados. O tempo médio de mensuração por parcela $(\bar{T})$ foi obtido por intermédio da divisão do tempo total de mensuração em cada dimensão de parcela, dividido pelo número total de unidades amostrais estabelecidas.

\section{Suficiência florística}

A partir de dados de riqueza de espécies plotados contra o número de indivíduos (densidade de espécies) foram produzidas curvas de rarefação adicionando-se as unidades amostrais em uma ordem aleatorizada 1.000 vezes, para todas as dimensões de parcelas. Após construir várias curvas, pode-se calcular uma curva do coletor média (baseada na riqueza média por unidade amostral) para cada dimensão de parcela. Em seguida obteve-se a média de todas estas estimativas para confecção das curvas de rarefação e configuração da possível variação em torno dessa média para cada dimensão de parcela (Gotelli \& Colwell, 2001).

Foram gerados e plotados intervalos de confiança para dois tipos de curvas que representam os menores e os maiores valores de riqueza observada, fornecendo padrões significativos de riqueza observada para todas as dimensões de parcelas (Gotelli \& Colwell, 2001).

No entanto, como as curvas de rarefação dificilmente constituem um platô, recomendase a utilização de uma estimativa como alternativa para obter-se a riqueza esperada de uma determinada área, e neste caso, aplicou-se o estimador Jackknife de primeira ordem para estimar a riqueza esperada para as diferentes dimensões de parcelas, no intuito de permitir a realização de uma estimativa confiável da riqueza (Colwell \& Coddington, 1994). Este estimador de riqueza é um método não-paramétrico que se baseia na proporção de espécies representadas por apenas um indivíduo.

Para avaliação da suficiência florística nas diferentes áreas avaliadas $\left(1.000 \mathrm{~m}^{2} ; 2.000 \mathrm{~m}^{2}\right.$; $4.000 \mathrm{~m}^{2} ; 10.000 \mathrm{~m}^{2}$ ), considerando a riqueza observada e a riqueza estimada calculada por intermédio do estimador Jackknife de primeira ordem, foi adotada a acurácia de 70\%, pois tal critério já foi utilizado em trabalhos anteriores (Meyer, 2015). Estas estimativas foram calculadas no software livre R versão 3.4.0 (R Core Team, 2017), por intermédio do pacote Vegan.

\section{RESULTADOS E DISCUSSÃO}

\section{Suficiência florística por dimensão de parcela}

Em relação ao número de famílias e espécies identificadas em cada dimensão de parcela selecionada, nas de $5 \mathrm{~m} \times 5 \mathrm{~m}$ foram catalogadas 29 famílias e 80 espécies, nas de $5 \mathrm{~m} \times 10 \mathrm{~m}$ foram 34 famílias e 103 espécies, nas $10 \mathrm{~m} \times 10 \mathrm{~m}$ foram 38 famílias e 123 espécies e em unidades amostrais de $10 \mathrm{~m} \times 25 \mathrm{~m}$ foram encontradas também 38 famílias e um total de 150 espécies.

O número de espécies por dimensão de parcela variou de 4 a 25 espécies nas de $5 \mathrm{~m} \times$ $5 \mathrm{~m}, 9$ a 27 nas de $5 \mathrm{~m} \times 10 \mathrm{~m}, 12$ a 33 espécies nas de $10 \mathrm{~m} \times 10 \mathrm{~m}$ e, 20 a 51 nas de $10 \mathrm{~m} \times 25 \mathrm{~m}$. Ou seja, a depender da dimensão de parcela selecionada, a variação do número de espécies pode ser 12 vezes maior que o inicial (4 a 51 espécies).

O aumento da riqueza de espécies proporcional ao aumento da dimensão de parcela selecionada já é esperado em estudos com espécies arbóreas regenerantes em fragmentos de florestas tropicais, a exemplo da Floresta Ombrófila Densa das Terras Baixas, pois não há ponto de estabilização da curva em termos do número de espécies que são observadas à medida que se aumenta o número de unidades amostrais e, caso inicialmente não fosse preestabelecido o número de parcelas, a área amostral total tenderia ao infinito (MuellerDombois \& Ellenberg, 1974). 
Normalmente, em florestas heterogêneas, por exemplo, Floresta Atlântica, a indicação de parcelas menores resulta no maior número de unidades amostrais distribuídas na área para se atingir o mesmo nível de precisão que em parcelas maiores, pois os coeficientes de variação decrescem à medida que se aumenta a dimensão das parcelas, para dada intensidade amostral (Sydow et al., 2017).

Compreende-se que ao desconsiderar o tempo de mensuração das análises, a dimensão de $10 \mathrm{~m} \times 25 \mathrm{~m}$ melhor representa a riqueza do fragmento, pois nesta dimensão de parcela são incluídas espécies exóticas e outras com baixa frequência que não foram registradas nas demais dimensões analisadas ( $5 \mathrm{~m} \times 5 \mathrm{~m} ; 5 \mathrm{~m} \times 10 \mathrm{~m}$ e $10 \mathrm{~m} \times 10 \mathrm{~m})$, acrescentando informações ecológicas relevantes para a conservação de espécies na área.

De maneira geral, à medida que o coeficiente de variação e o erro amostral diminuem quando se aumenta a dimensão de parcela, os erros não-amostrais tendem a aumentar (Meunier et al., 2001). Ou seja, quanto maior for a unidade amostral, maior será possibilidade de se cometer erros não-amostrais tendo em vista às dificuldades na supervisão do trabalho de campo e repetitivas mensurações.

Ressalta-se que para selecionar a dimensão ideal de parcela em levantamentos fitossociológicos na Floresta Atlântica deve-se atentar não apenas a critérios puramente estatísticos, mas também a informações ecológicas inerentes a área, tais como número de espécies.

Em estudos com abordagem fitossociológica, o número de espécies é o atributo mais (importante) utilizado para descrever a comunidade, uma vez que permite fornecer uma informação segura da diversidade de espécies (Townsend et al., 2010). Neste sentido, a escolha da dimensão de parcelas e do erro amostral admissível em dado nível de probabilidade podem ser determinantes para obtenção resultados mais satisfatórios.

As curvas de rarefação plotadas apesar de indicarem elevada diferença de riqueza específica entre as comunidades analisadas, não assumiram a assíntota em nenhuma das dimensões de parcelas adotadas (Figura 2), sendo possível prever um considerável incremento de espécies, caso a abrangência da área amostral aumente.

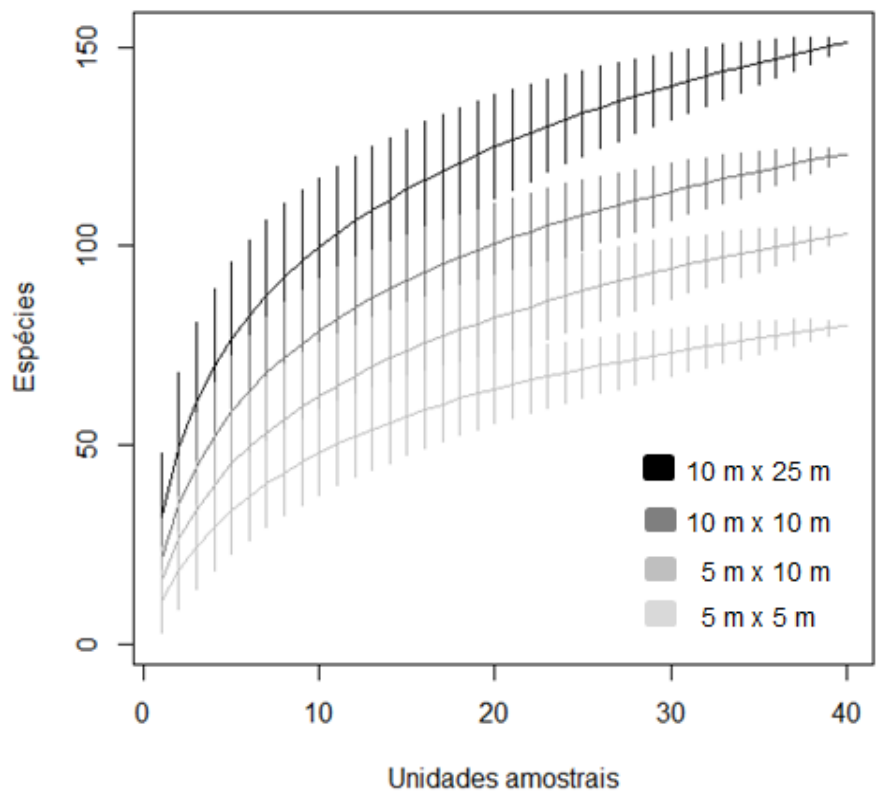

Figura 2. Curva de acumulação espécie/área por dimensões de parcelas ( $5 \mathrm{~m} \times 5 \mathrm{~m}$ - cor cinza claro; $5 \mathrm{~m}$ x $10 \mathrm{~m}$ - cor cinza; $10 \mathrm{~m} \times 10 \mathrm{~m}$ - cor cinza escuro, $10 \mathrm{~m} \times 25 \mathrm{~m}$ - cor preta) pelo método de rarefação (aleatorizado 1.000 vezes).

De acordo com o estimador não-paramétrico Jackknife de primeira ordem, obteve-se a estimativa de 103 espécies para as parcelas com dimensões de $5 \mathrm{~m} \times 5 \mathrm{~m}\left(1.000 \mathrm{~m}^{2}\right)$, 
133 espécies para as de $5 \mathrm{~m} \times 10 \mathrm{~m}\left(2.000 \mathrm{~m}^{2}\right), 155$ espécies para as de $10 \mathrm{~m} \times 10 \mathrm{~m}\left(4.000 \mathrm{~m}^{2}\right) \mathrm{e}$ 185 espécies para as de $10 \mathrm{~m} \times 25 \mathrm{~m}\left(10.000 \mathrm{~m}^{2}\right)$.

Os resultados indicados pelo estimador Jackniffe de primeira ordem evidenciam que foram inventariadas para cada uma das dimensões de parcelas analisadas, respectivamente, $77 \%, 77 \%, 79 \%$ e $81 \%$ das espécies estimadas. Sendo a acurácia superior a $70 \%$ entre as duas medidas (riqueza observada "versus" riqueza estimada), indicativo que as amostragens foram suficientemente representativas (Meyer, 2015).

\section{Suficiência amostral para número de indivíduos}

De acordo com o critério de erro amostral adotado, pode-se inferir que as dimensões de parcela de $5 \mathrm{~m} \times 5 \mathrm{~m}(E A=12,5 \% ; p=63)$ e $5 \mathrm{~m} \times 10 \mathrm{~m}(E A=11,5 \% ; p=54)$ não atendem a suficiência amostral nem tampouco o erro amostral preconizado (Quadro 1), sendo portanto desconsideradas no cálculo da eficiência relativa por não atingirem níveis de comparação, em termos de número de indivíduos amostrados com as demais dimensões analisadas. Tornando, neste caso, recomendadas apenas comparações entre as parcelas de $10 \mathrm{~m} \times 10 \mathrm{~m}$ $(E A=9,8 \% ; p=39)$ e $10 \mathrm{~m} \times 25 \mathrm{~m}(E A=7,9 \% ; p=25)$ (Quadro 1).

Quadro 1. Principais estimadores para número de indivíduos arbóreos regenerantes por dimensões de parcelas ( $5 \mathrm{~m} \times 5 \mathrm{~m} ; 5 \mathrm{~m} \times 10 \mathrm{~m} ; 10 \mathrm{~m} \times 10 \mathrm{~m} ; 10 \mathrm{~m} \times 25 \mathrm{~m})$. CV = Coeficiente de variação; EA = Erro amostral; N.A.S. = Número de amostras suficientes (p); A.A.S. = Área amostral suficiente $\left(\mathrm{m}^{2}\right)$.

\begin{tabular}{|cccccccc|}
\hline & \multicolumn{3}{c}{$\mathbf{t}_{(\mathbf{0}, \mathbf{0 5} ; \mathbf{3 9 G})=\mathbf{1 , 6 8 4 8 7 5}}$} & \multicolumn{2}{c|}{ EA admissível $=\mathbf{1 0 \%}$} \\
\hline $\begin{array}{c}\text { Dimensões } \\
\text { de parcelas }\end{array}$ & $\begin{array}{c}\mathbf{N}^{\mathbf{0}} \text { total } \\
\text { de } \\
\text { indivíduos }\end{array}$ & Média & $\begin{array}{c}\text { Intervalo de } \\
\text { confiança } \\
\text { para a média }\end{array}$ & CV (\%) & EA (\%) & NAS $\left(\mathbf{p}_{\mathbf{1 0}}\right)$ & AAS $\left(\mathbf{m}^{\mathbf{2}}\right)$ \\
\hline $5 \mathrm{~m} \times 5 \mathrm{~m}$ & 1.028 & 25,7 & $25,7 \pm 3,2$ & 46,9 & 12,5 & 63 & 1.575 \\
\hline $5 \mathrm{~m} \times 10 \mathrm{~m}$ & 2.040 & 51 & $51 \pm 5,9$ & 43,3 & 11,5 & 54 & 2.700 \\
\hline $10 \mathrm{~m} \times 10 \mathrm{~m}$ & 4.009 & 100,2 & $100,2 \pm 9,9$ & 36,6 & 9,8 & 39 & 3.900 \\
\hline $10 \mathrm{~m} \times 25 \mathrm{~m}$ & 9.285 & 232,1 & $232,1 \pm 18,5$ & 29,6 & 7,9 & 25 & 6.250 \\
\hline
\end{tabular}

Considerando o número de indivíduos amostrados, observa-se que o ponto de máxima curvatura estabelecido para o limite de erro aceitável de 10\% com nível de probabilidade de $95 \%$, indica que ao selecionar a dimensão de $5 \mathrm{~m} \times 5 \mathrm{~m}$ ou $5 \mathrm{~m} \times 10 \mathrm{~m}$ obtêm-se considerável redução do tempo médio de mensuração dos indivíduos (custo) em contrapartida há um considerável aumento do erro amostral em relação às demais dimensões analisadas. Contrariamente, com a utilização da dimensão de parcela de $10 \mathrm{~m} \times 25 \mathrm{~m}$ adquire-se o menor erro amostral, mas registra-se o maior tempo médio de mensuração em relação às demais (Figura 3).

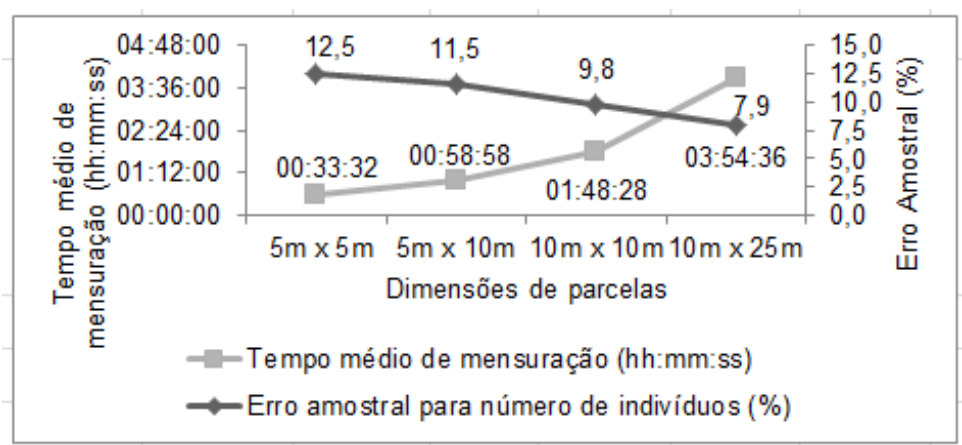

Figura 3. Ponto de máxima curvatura relacionando o erro amostral para número de indivíduos amostrados (em \%) e o tempo médio de mensuração dos indivíduos por unidade amostral (hh:mm:ss) às dimensões de parcelas $(5 \mathrm{~m} \times 5 \mathrm{~m} ; 5 \mathrm{~m} \times 10 \mathrm{~m} ; 10 \mathrm{~m} \times 10 \mathrm{~m}$ e $10 \mathrm{~m} \times 25 \mathrm{~m})$. (L.E. $=10 \% ; \mathrm{t}(0,05 ; 39 \mathrm{GL})=$ $1,684875)$. 
Ainda considerando o número de indivíduos registrados, sob o critério de erro amostral adotado e o tempo médio de mensuração do inventário, o ponto de intersecção das curvas aponta que $10 \mathrm{~m} \times 10 \mathrm{~m}$ é a dimensão ideal de parcela (Figura 2), tendo a elevada precisão amostral com baixo tempo médio e total de mensuração, respectivamente.

Eficiência relativa para número de indivíduos

Reitera-se que as dimensões de parcelas de $5 \mathrm{~m} \times 5 \mathrm{~m}$ e $5 \mathrm{~m} \times 10 \mathrm{~m}$ foram desconsiderados da análise da eficiência relativa por não atenderem a suficiência amostral para número de indivíduos com erro admissível de $10 \%$ e $95 \%$ de probabilidade.

Os resultados apontam que a dimensão de parcelas de $10 \mathrm{~m} \times 10 \mathrm{~m}$ é a mais eficiente $(E R=100 \%)$ para representar a área estudada, considerando o tempo de mensuração do inventário, o erro amostral preconizado de $10 \%$ para número de indivíduos amostrados e a precisão dos dados obtidos quando comparado com a dimensão de parcela de $10 \mathrm{~m} \times 25 \mathrm{~m}$ que apresentou eficiência relativa de $25 \%$ apenas.

\section{Suficiência amostral para número de espécies}

Conforme o critério de erro amostral preestabelecido, pode-se concluir que a dimensão de parcela de $5 \mathrm{~m} \times 5 \mathrm{~m}(\mathrm{EA}=10,1 \% ; p=42)$ não atingiu a suficiência amostral (Quadro 2), sendo portanto desconsiderada no cálculo da eficiência relativa por não atingir nível de comparação, em termos do número de espécies amostradas com as dem ais dimensões analisadas. Sendo, sob estes critérios, apenas recomendadas comparações com as parcelas de $5 \mathrm{~m} \times 10 \mathrm{~m}$ $(E A=7,7 \% ; p=24), 10 \mathrm{~m} \times 10 \mathrm{~m}(E A=6,2 \% ; p=16)$ e $10 \mathrm{~m} \times 25 \mathrm{~m}(E A=6,9 \% ; p=20)$ (Quadro 2).

Quadro 2. Principais estimadores para número de espécies arbóreas regenerantes amostradas por dimensões de parcelas ( $5 \mathrm{~m} \times 5 \mathrm{~m} ; 5 \mathrm{~m} \times 10 \mathrm{~m} ; 10 \mathrm{~m} \times 10 \mathrm{~m} ; 10 \mathrm{~m} \times 25 \mathrm{~m})$. CV = Coeficiente de variação; $E A=$ Erro amostral; N.A.S. = Número de amostras suficientes (p); A.A.S. $=$ Área amostral suficiente $\left(\mathrm{m}^{2}\right)$.

\begin{tabular}{|c|c|c|c|c|c|c|c|}
\hline \multirow[b]{2}{*}{$\begin{array}{l}\text { Dimensões } \\
\text { de parcelas }\end{array}$} & \multirow[b]{2}{*}{$\begin{array}{l}\mathbf{N}^{\circ} \text { total } \\
\text { de } \\
\text { espécies }\end{array}$} & \multicolumn{4}{|c|}{$t_{(0,05 ; 39 G L)}=1,684875$} & \multicolumn{2}{|c|}{ EA admissível = $10 \%$} \\
\hline & & Média & $\begin{array}{l}\text { Intervalo de } \\
\text { confiança } \\
\text { para a } \\
\text { média }\end{array}$ & CV (\%) & EA (\%) & NAS $\left(p_{10}\right)$ & $\operatorname{AAS}\left(\mathrm{m}^{2}\right)$ \\
\hline $5 \mathrm{~m} \times 5 \mathrm{~m}$ & 80 & 10,6 & $10,6 \pm 1,1$ & 38,0 & 10,1 & 42 & 1.050 \\
\hline $5 \mathrm{~m} \times 10 \mathrm{~m}$ & 103 & 15,5 & $15,5 \pm 1,2$ & 29,0 & 7,7 & 24 & 1.200 \\
\hline $10 \mathrm{~m} \times 10 \mathrm{~m}$ & 123 & 21,5 & $21,5 \pm 1,3$ & 23,3 & 6,2 & 16 & 1.600 \\
\hline $10 \mathrm{~m} \times 25 \mathrm{~m}$ & 150 & 32,1 & $32,1 \pm 2,2$ & 25,8 & 6,9 & 19 & 4.750 \\
\hline
\end{tabular}

O ponto de intersecção das curvas do erro amostral admissível de 10\% (com probabilidade de $95 \%$ ) e do tempo médio de mensuração do inventário aponta que $10 \mathrm{~m} x$ $10 \mathrm{~m}$ é a dimensão ideal de parcela a ser utilizada neste estudo (Figura 4), apresentando elevada precisão amostral (erro de amostragem para número de espécies abaixo de 10\%) com baixo tempo médio e total de mensuração, respectivamente.

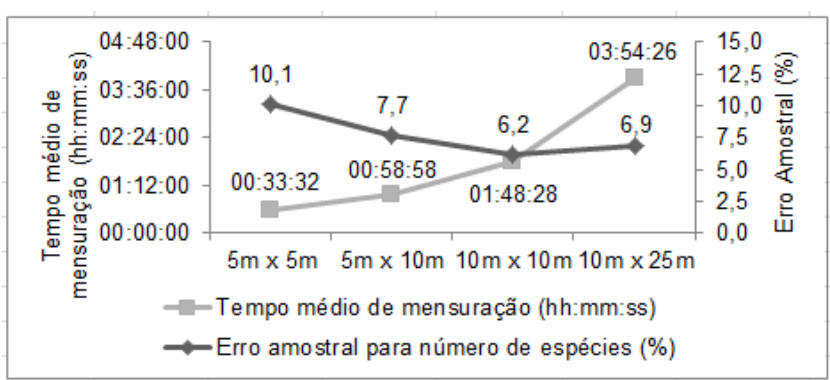

Figura 4. Ponto de máxima curvatura relacionando o erro de amostragem para número de espécies (em \%) e o tempo médio de mensuração dos indivíduos por unidade amostral (hh:mm:ss) às dimensões de parcelas $(5 \mathrm{~m} \times 5 \mathrm{~m} ; 5 \mathrm{~m} \times 10 \mathrm{~m} ; 10 \mathrm{~m} \times 10 \mathrm{~m}$ e $10 \mathrm{~m} \times 25 \mathrm{~m})$. $\left(\right.$ L.E. $\left.=10 \% ; \mathrm{t}_{(0,05 ; 39 \mathrm{GL})}=1,684875\right)$. 


\section{Eficiência relativa para número de espécies}

Reitera-se que a dimensão de parcelas de $5 \mathrm{~m} \times 5 \mathrm{~m}$ foi desconsiderada da análise da eficiência relativa por não atender a suficiência amostral com erro admissível de 10\% e 95\% de probabilidade.

Os resultados indicam que a dimensão de $10 \mathrm{~m}$ x 10m é a mais eficiente (ER > 100\%) para representar a área estudada, considerando o tempo médio e total de mensuração dos indivíduos (custos do inventário), o erro amostral adotado de $10 \%$ e a precisão dos dados obtidos quando comparado com as dimensões de $5 \mathrm{~m} \times 10 \mathrm{~m}$ ( $E R=100 \%$ ) e de $10 \mathrm{~m} \times 25 \mathrm{~m}$ que apresentou ER de 35\%.

Adicionalmente aos resultados da análise da eficiência relativa para número de indivíduos e de espécies, destaca-se a elevada representatividade de (123) espécies registradas na dimensão de $10 \mathrm{~m} \times 10 \mathrm{~m}$, tendo em vista a relevância desta informação ecológica para melhor compreender sobre as variações florísticas e estruturais das espécies arbóreas regenerantes no fragmento da Mata de Manassu.

Apesar de não haver consenso quanto à dimensão e número de parcelas a serem estabelecidas em pesquisas com a regeneração natural de espécies arbóreas no bioma Mata Atlântica, os resultados apontam decréscimo na caracterização da riqueza de espécies ao se adotar parcelas muito pequenas, ou mesmo ao se incluir critérios de erro amostral admissível superior a $10 \%$ para o fragmento florestal analisado.

\section{CONCLUSÕES}

A amostragem da vegetação arbórea regenerante na Mata de Manassu com diferentes dimensões de parcelas apresenta distintos níveis de riqueza de espécies e de precisão por tempo de amostragem e indica que a dimensão de $10 \mathrm{~m}$ x $10 \mathrm{~m}$ é mais eficiente para realização deste estudo, dada à elevada precisão amostral, alta riqueza de espécies e o baixo de custo de amostragem.

Este estudo aponta decréscimo na caracterização da riqueza de espécies arbóreas regenerantes ao se adotar parcelas com dimensões iguais ou inferiores a $5 \mathrm{~m} \times 10 \mathrm{~m}$, ou mesmo ao se incluir critérios de erro amostral admissível superior a 10\% para o fragmento florestal analisado, e possibilita melhor direcionamento quanto ao planejamento de futuros trabalhos a serem desenvolvidos com a regeneração natural de espécies arbóreas em fragmentos de Floresta Ombrófila Densa das Terras Baixas no Estado de Pernambuco.

Desta forma, recomenda-se a realização de análises semelhantes em futuros trabalhos que envolvam a fitossociologia de espécies arbóreas regenerantes em fragmentos de Floresta Ombrófila Densa das Terras Baixas. Caso não seja possível adotar tais análises em função de custos, preferencialmente propõe-se adotar critérios estatísticos mais acurados com relação à precisão, tais como erro amostral admissível igual ou inferior a $10 \%$ com nível de confiabilidade de 95\%, no intuito de se obter informações ecológicas mais fidedignas a respeito das variações florístico-estruturais de espécies na área a ser estudada.

\section{AGRADECIMENTOS}

A Universidade Federal Rural de Pernambuco (UFRPE), em especial ao Programa de PósGraduação em Ciências Florestais (PPGCF) pela oportunidade concedida e a Coordenação de Aperfeiçoamento de Pessoal de Nível Superior (CAPES) pela concessão da bolsa de estudos durante a realização da pesquisa.

\section{REFERÊNCIAS BIBLIOGRÁFICAS}

Alvares, C. A., Stape, J. L., Sentelhas, P. C., \& Gonçalves, J. L. M. (2013). Modeling monthly mean air temperature for Brazil. Theoretical and Applied Climatology, 113(3-4), 407-427. http://dx.doi.org/10.1007/s00704-012-0796-6.

Agência Pernambucana de Águas e Clima - APAC. (2017). Monitoramento Pluviométrico. Visualização mensal. Data inicial: 01 de julho de 2016 - Data final: 30 de junho de 2017. Mesorregião: Metropolitana 
do Recife. Posto: Jaboatão dos Guararapes (Barragem Duas Unas) (268). Recuperado em 27 de junho de 2018, de http://www.apac.pe.gov.br/meteorologia/monitoramento-pluvio.php?posto_id=268

Angiosperm Phylogeny Group IV - APG IV. (2016). An update of the Angiosperm Phylogeny Group classification for the orders and families of flowering plants: APG IV. Botanical Journal of the Linnean Society, 181(1), 1-20. https://doi.org/10.1111/boj.12385.

Bonetes, L. (2003). Tamanho de parcelas e intensidade amostral para estimar o estoque e índices fitossociológicos em uma Floresta Ombrófila Mista. 111 f. (Dissertação de mestrado). Universidade Federal do Paraná, Curitiba.

Colwell, R., \& Coddington, J. A. (1994). Estimating terrestrial biodiversity through extrapolation. Philos. Trans. R. Soc. London B series, 345(1311), 101-118. Recuperado em 13 de janeiro de 2018, de https://repository.si.edu/bitstream/handle/10088/4441/Colwell_Coddington94.pdf?sequence=1\&isA llowed=y

Gomes, J. S., Lins-e-Silva, A. C. B., Rodal, M. J. N., \& Silva, H. C. H. (2009). Estrutura do sub-bosque lenhoso em ambientes de borda e interior de dois fragmentos de Floresta Atlântica em Igarassu, Pernambuco, Brasil. Rodriguésia, 60(2), 295-310.

Gotelli, N. J., \& Colwell, R. K. (2001). Quantifying biodiversity: procedures and pitfalls in the measurement and comparison of species richness. Ecology Letters, 4(i.4), 379-391. Recuperado em 03 de setembro de 2018, de http://onlinelibrary.wiley.com/doi/10.1046/j.14610248.2001.00230.x/epdf

Higuchi, N., Santos, J., \& Jardim, F. C. S. (1982). Tamanho de parcela amostral para inventários florestais. Acta Amazonica, 12(1), 91-103. Recuperado em 02 de agosto de 2018, de https://acta.inpa.gov.br/fasciculos/12-1/PDF/v12n1a13.pdf

Lima, R. B. A. (2017). Estrutura, regeneração natural e ciclagem de nutrientes em Floresta Ombrófila Densa das Terras Baixas, na Zona da Mata de Pernambuco. 150 f. (Tese de doutorado). Universidade Federal Rural de Pernambuco, Recife. Recuperado em 21 de setembro de 2018, de http://www.ppgcf.ufrpe.br/sites/www.ppgcf.ufrpe.br/files/documentos/rosival_barros_de_andrade_ lima_0.pdf

Meunier, I. M. J., Silva, J. A. A., \& Ferreira, R. L. C. (2001). Inventário florestal: Programas de estudo (179 p.). Recife, PE: Universidade Federal Rural de Pernambuco.

Meyer, L. (2015). Suficiência amostral e preditores ambientais da vegetação de sub-bosque da Mata Atlântica de Santa Catarina, Brasil. 88 f. (Dissertação de mestrado). Universidade Federal de Minas Gerais, Belo Horizonte. Recuperado em 21 de janeiro de 2018, de http://www.bibliotecadigital.ufmg.br/dspace/bitstream/handle/1843/BUOS9WMTLK/disserta_o_vers_o_final_leila_meyer.pdf?sequence $=1$

Mueller-Dombois, D., \& Ellenberg, H. (1974). Aims and methods of vegetation ecology (547 p.). New York: John Wiley \& Sons.

Oliveira, L. S. C., Marangon, L. C., Feliciano, A. L. P., Lima, A. S., Cardoso, M. S. O., \& Santos, W. B. (2015). Efeito de borda em remanescentes de Floresta Atlântica na Bacia do Rio Tapacurá, Pernambuco. Cerne, 21(2), 169-174. Recuperado em 18 de agosto de 2018, de http://www.scielo.br/pdf/cerne/v21n2/2317-6342-cerne-21-02-00169.pdf

Péllico Netto, S., \& Brena, D. A. (1997). Inventário florestal (Vol. 1, 316 p.). Curitiba: UFPR.

R Core Team. (2017). R: A language and environment for statistical computing (version 3.4.0). Vienna, Austria: R Foundation for Statistical Computing. Recuperado em 15 de julho de 2018, de https://www.R-project.org/

Rode, R., Figueiredo Filho, A., Machado, A. S., \& Galvão, F. (2010). Análise do padrão espacial de espécies e de grupos florísticos estabelecidos em um povoamento de Araucaria angustifolia em uma Floresta Ombrófila Mista no Centro-Sul do Paraná. Floresta, 40(2), 255-268. Recuperado em 17 de outubro de 2018, de http://revistas.ufpr.br/floresta/article/view/17821/11623

Silva, F. B. R., Santos, J. C. P., Silva, A. B., Cavalcanti, A. C., Silva, F. H. B. B., Burgos, N., Parahyba, R. B. V., Oliveira Neto, M. B., Sousa Neto, N. C., Araújo Filho, J. C., Lopes, O. F., Luz, L. R. P. P., Leite, A. P., Souza, L. G. M. C., Silva, C. P., Varejão-Silva, M. A., \& Barros, A. H. C. (2001). Zoneamento Agroecológico do Estado de Pernambuco (Embrapa Solos. Documentos, 35, 1 CD ROM). Recife: Embrapa Solos-UEP Recife.

Silva, R. K. S. (2017). Dinâmica e relação solo-vegetação do componente arbustivo-arbóreo da regeneração natural em fragmento de floresta Atlântica, Pernambuco. 149 f. (Tese de doutorado). Universidade Federal Rural de Pernambuco, Recife. Recuperado em 04 de fevereiro de 2018, de 
http://www.ppgcf.ufrpe.br/sites/www.ppgcf.ufrpe.br/files/documentos/roseane_karla_soares_da_sil va_0.pdf

Silva, W. C., Marangon, L. C., Ferreira, R. L. C., Feliciano, A. L. P., Aparício, O. S., \& Costa Junior, R. F. (2010). Estrutura horizontal e vertical do componente arbóreo em fase de regeneração natural na mata Santa Luzia, no município de Catende-PE. Revista Árvore, 34(5), 863-869. Recuperado em 25 de agosto de 2018, de http://www.scielo.br/scielo.php?script=sci_arttext\&pid=S010067622010000500011

Soares, C. P. B., Paula Neto, F., \& Souza, A. L. (2011). Dendrometria e inventário florestal (2. ed.). Viçosa: Editora UFV. 272p.

Sydow, J. D., Sanquetta, C. R., \& Corte, A. P. D. (2017). Comparação de métodos e processos de amostragem para inventário em Floresta Ombrófila Mista. Biofix Scientific Journal, 2(1), 60-68. Recuperado em 22 de setembro de 2018, de http://revistas.ufpr.br/biofix/article/view/50761/32281.

Torres, J. E. L. (2014). Espécies arbóreas regenerantes na borda e no interior de um fragmento de floresta atlântica em Pernambuco. 61 f. (Dissertação de Mestrado). Universidade Federal Rural de Pernambuco, Recife. Recuperado em 25 de agosto de 2018, de http://www.ppgcf.ufrpe.br/sites/www.ppgcf.ufrpe.br/files/documentos/jose_edson_de_lima_torres_ 0.pdf

Townsend, C. R., Begon, M., \& Harper, J. L. (2010). Fundamentos em ecologia (3. ed., 592 p.). Porto Alegre: Artmed.

Vasconcelos, A. J. N. (1990). Otimização de parcelas em levantamentos botânicos em áreas de solos brunos não cálcicos do Estado de Pernambuco.106 f. (Dissertação de mestrado). Universidade Federal Rural de Pernambuco, Recife.

Contribuição dos Autores: NDS: Conceituação, Curadoria de Dados, Análise Formal, Investigação, Metodologia, Recursos, Visualização, Escrita - Primeira Redação, Escrita - Revisão e Edição; LCM: Conceituação, Curadoria de Dados, Análise Formal, Obtenção de Financiamento, Metodologia, Administração do Projeto, Recursos, Supervisão, Validação, Visualização, Escrita - Primeira Redação, Escrita - Revisão e Edição; ALFPM e JAAS: Conceituação, Curadoria de Dados, Análise Formal, Metodologia, Recursos, Supervisão, Validação, Visualização, Escrita - Primeira Redação, Escrita - Revisão e Edição. 